\title{
Influence of the Carotenoid Composition on the Conformational 2 Dynamics of Photosynthetic Light-Harvesting Complexes
}

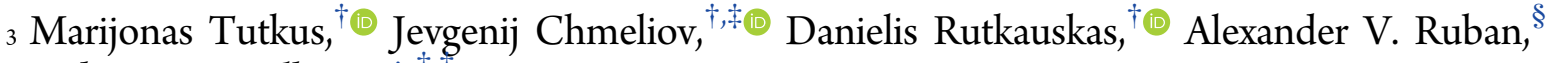 \\ 4 and Leonas Valkunas $*,+$, \\ $5{ }^{\dagger}$ Department of Molecular Compound Physics, Center for Physical Sciences and Technology, Sauletekio Avenue 3, LT-10257 Vilnius, \\ 6 Lithuania

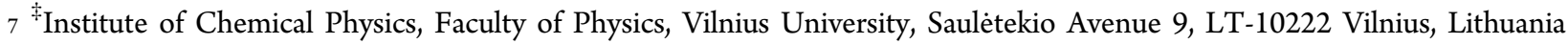 \\ $8{ }^{\S}$ The School of Biological and Chemical Sciences, Queen Mary, University of London, Mile End Road, London E1 4NS, United \\ 9 Kingdom
}

10 S Supporting Information

11 ABSTRACT: Nonphotochemical quenching (NPQ) is the major self-regulatory 12 mechanism of green plant, performed on a molecular level to protect them from an 13 overexcitation during the direct sunlight. It is believed that NPQ becomes available due to 14 conformational dynamics of the light-harvesting photosynthetic complexes and involves a 15 direct participation of carotenoids. In this work, we perform a single-molecule microscopy 16 on major light-harvesting complexes (LHCII) from different Arabidopsis thaliana mutants 17 exhibiting various carotenoid composition. We show how the distinct carotenoids affect the 18 dynamics of the conformational switching between multiple coexisting light-emitting states 19 of LHCII and demonstrate that properties of the quenched conformation are not 20 influenced by the particular carotenoids available in LHCII. We also discuss the possible 21 origin of different conformational states and relate them to the fluorescence decay kinetics 22 observed during the bulk measurements.

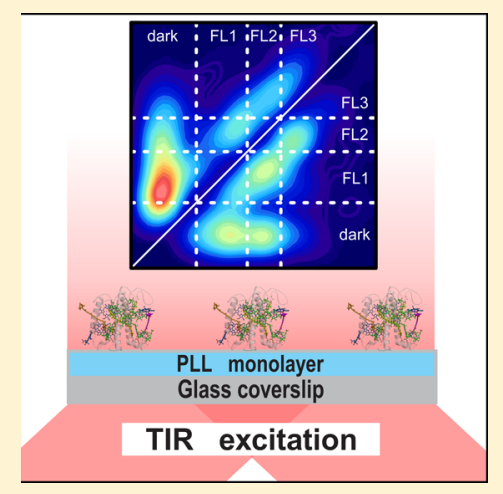

${ }_{23} \pi$ he importance of photosynthesis and its role in the Earth's 24 ecosystem is difficult to overestimate: it is the key 25 physiological process performed by green plants, algae, and 26 some bacteria that is responsible for the initial step of biomass 27 production and refilling the atmosphere with oxygen. To 28 efficiently perform this task, distinct photosynthetic organisms 29 have developed various photosynthetic apparatuses different in 30 structure but similar in design. Their "photosynthetic factories" 31 comprise a huge number of pigment molecules, usually bound 32 to a protein scaffold and distributed over a photosynthetic 33 membrane. ${ }^{1,2}$ Both the mutual arrangement of the pigments 34 and their spectroscopic properties have been carefully adjusted 35 by Nature to optimize an overall efficiency of the photo36 synthetic light-harvesting antenna: up to $99 \%$ of the absorbed 37 photons are successfully utilized during the later stages of 38 photosynthesis. $^{1-4}$ While such an efficiency helps photo39 synthetic organisms to survive and to function at very low 40 illumination conditions (like underwater environment or deep 41 continuous shadow), bright sunlight might result in an 42 overexcitation of the light-harvesting antenna and lead to the 43 formation of highly reactive singlet oxygen species. In order to 44 avoid any possible photodamage, over ages of evolution, plants 45 have developed various self-regulatory mechanisms. The most 46 efficient one, operating on a molecular level and dissipating the 47 excess excitation as heat, reversibly forms and relaxes within 48 several seconds to minutes and is commonly known as an 49 energy-dependent ( $\mathrm{qE}$ ) part of nonphotochemical quenching
(NPQ). ${ }^{5}$ Many studies aiming to reveal the molecular origin of 50 NPQ have been carried out over the last two decades (see, e.g., 51 the recent review by Ruban et al. ${ }^{5}$ ), but the final answer is still 52 to be found, although more evidence for the direct involvement 53 of the carotenoid (Car) molecules appears. ${ }^{6-11} 54$

The larger part of the photosynthetic antenna of plants is 55 built from the trimeric major light-harvesting complexes 56 (LHCIIs) that bind more than $50 \%$ of all the terrestrial 57 chlorophyll (Chl) molecules. ${ }^{1}$ These complexes, however, are 58 known not only for the efficient light harvesting, but also for 59 participation in various regulatory processes. First, depending 60 on the spectral composition of the incoming light, LHCII 61 trimers can diffuse through the thylakoid membrane between 62 different photosynthetic units to optimize their relative 63 absorption cross sections. ${ }^{12}$ Besides that, LHCII complexes 64 participate in the dynamic variation of the antenna size during 65 high light conditions ${ }^{13}$ and are also supposed to be the most 66 probable location for the NPQ traps. ${ }^{5}$

The crystal structure of the LHCII complexes, known with a 68 sub-3 A resolution, ${ }^{14}$ reveals the mutual arrangement of $8 \mathrm{Chl} a 69$ and $6 \mathrm{Chl} b$ molecules as well as 4 Car pigments: 2 luteins 70 (Lut), 1 neoxanthin (Neo), and 1 xanthophyll cycle carotenoid 71 (either violaxanthin (Vio) or zeaxanthin (Zea)) per each $72 \mathrm{fl}$

Received: October 5, 2017

Accepted: November 15, 2017

Published: November 15, 2017 
f1 73 monomeric subunit (see Figure 1a for schematic view). Neo, 74 the most polar and asymmetric of these Cars, is found on a Chl
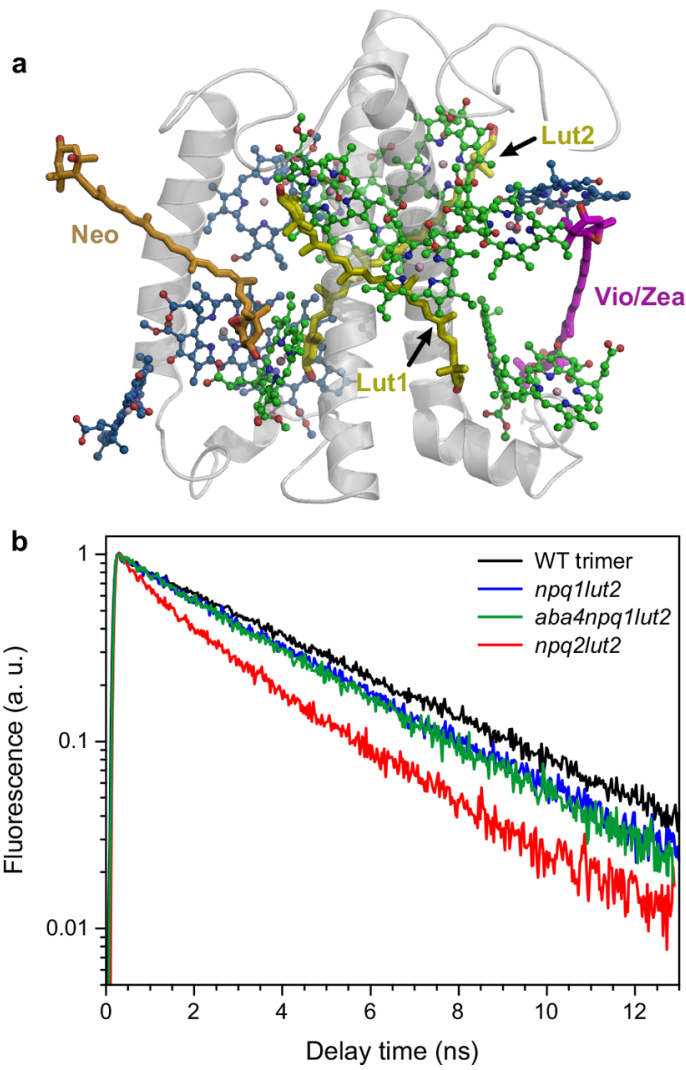

Figure 1. (a) Crystal structure of LHCII monomer and carotenoid binding sites (Lut1, Lut2, Vio, and Neo/Zea). ${ }^{14}$ Chls $a$ are indicated with green, Chls $b$ with blue, both luteins with dark yellow, Vio with magenta, Neo with orange, and protein helices with gray. (b) Fluorescence decay kinetics in the detergent-solubilized LHCII complexes from WT Arabidopsis as well as npq1lut2 (containing Vio and Neo carotenoids), aba4 npq1lut2 (binding Vio as the only Car), and npq2lut2 (binding Zea as the only Car) mutants, measured at 273 $\mathrm{K}$.

$75 b$-rich peripheral side of LHCII. The xanthophyll cycle Car is 76 located at the interface between the two monomeric subunits of 77 the LHCII trimer. Exposure to high light results in a reversible 78 de-epoxidation of Vio into Zea, which promotes the clustering 79 of the LHCII complexes, leading to a more efficient excitation 80 energy quenching. ${ }^{5}$ Finally, the remaining two Cars, luteins, are 81 arranged in a cross pattern and assist in holding the LHCII 82 complex together. ${ }^{14}$ This Car is also known to be responsible 83 for the trimerization of $\mathrm{LHCII}^{15}$ therefore lutein-deficient 84 plants are able to form only monomeric light-harvesting 85 complexes. ${ }^{16}$ Moreover, one of these luteins, namely Lut 1 , is 86 located close to the so-called chlorophyll terminal emitter the 87 cluster of $3 \mathrm{Chl}$ molecules of the lowest site energies, which 88 makes it a favorable candidate to govern NPQ. ${ }^{8}$

89 To study the role of each carotenoid in light harvesting and 90 self-regulation, the targeted mutagenesis of various photo91 synthetic antenna complexes has been widely applied. ${ }^{6,16-22}$ By 92 blocking the specific paths of Car biosynthesis, LHCIIs with 93 different xanthophyll composition can be obtained, causing 94 specific structural variations that affect the overall excitation 95 energy dynamics in the light-harvesting antenna. ${ }^{16}$ In this work, 96 we utilize the methods of single molecule (SM) microscopy to examine fluorescence (FL) intensity fluctuations in single 97 monomeric LHCII subunits from the lutein-deficient Arabi- 98 dopsis thaliana mutants. In particular, we study the double 99 mutant npq1lut $2,{ }^{17}$ which is incapable of synthesizing either Zea 100 or Lut and therefore accumulates only Vio and Neo, and the 101 triple mutants aba4npq1lut2 ${ }^{20}$ and npq2lut2, ${ }^{23}$ accumulating 102 Vio and Zea, respectively, as the only carotenoid. The Car 103 composition of these mutants is also summarized in Table 1. $104 \mathrm{tl}$ For reference, isolated trimeric LHCII complexes from 105 unaffected wild-type (WT) Arabidopsis thaliana were also 106 analyzed.

Table 1. Carotenoid Composition in the LHCII Samples Studied in This Work

\begin{tabular}{lc}
\multicolumn{1}{c}{ sample } & bound carotenoids \\
WT & Lut, Vio, and Neo \\
npq1lut2 & Vio and Neo \\
aba4npq1lut2 & Vio \\
npq2lut2 & Zea
\end{tabular}

Fluorescence decay kinetics in the mentioned bulk 108 solubilized LHCII samples, measured with a streak-camera at 109 $273 \mathrm{~K}$ temperature, are shown in Figure $1 \mathrm{~b}$. Those in all 110 mutants clearly demonstrate somewhat faster decay behavior 111 compared to the WT species. Thus, replacement of the lutein 112 pigments by the xanthophyll cycle carotenoid (either Vio or 113 Zea) possibly opens some additional weak channel for 114 excitation quenching, not available in wild-type LHCIIs. Since 115 Neo is located in the Chl $b$-rich region of the LHCII monomer 116 (cf. Figure 1a), its replacement by the Vio pigment in the 117 aba4npq1lut2 mutant does not influence the mean relaxation 118 rate of the Chls $a$ (mean excitation lifetimes in the npq1lut2 and 119 aba4npq1lut2 mutants are 3.3 and $3.2 \mathrm{~ns}$, respectively). 120 Meanwhile, npq2lut2 mutant, incapable of synthesizing any 121 carotenoid except Zea, shows huge qualitative difference in the 122 fluorescence decay kinetics, clearly exhibiting a strongly 123 biexponential decay behavior contrary to all three of the 124 other samples, demonstrating almost single-exponential decay 125 (see Table S1 for lifetimes and their amplitudes). The 126 appearance of the additional fast decay component of $0.8 \mathrm{~ns} 127$ strongly suggests that a new conformational state of the protein 128 scaffold, notably enhancing excitation quenching, becomes 129 available in this mutant. To perform further analysis of the 130 intrinsic conformational states and their dynamics, we 131 continued with SM microscopy measurements of single 132 LHCII complexes, immobilized on the PLL modified glass 133 coverslips in detergent micelles.

134

For our measurements, we used total internal reflection 135 (TIR) objective-based microscope setup equipped with the 136 EM-CCD camera working at $30 \mathrm{~ms}$ integration time and 635137 nm laser excitation. ${ }^{24}$ This method allowed us to subject LHCII 138 complexes to the continuous $<1 \mathrm{~W} / \mathrm{cm}^{2}$ illumination, which is 139 considerably lower than typical intensities utilized in confocal 140 microscopy, ${ }^{25-30}$ thus we completely avoid any singlet singlet 141 and singlet triplet annihilation (taking into account the 142 absorption cross-section of an LHCII trimer of $\sigma=1.4 \times 143$ $10^{-15} \mathrm{~cm}^{2}$, 31 the probability of simultaneous generation of two 144 singlet excited states is negligible, and the probability of 145 accumulating a triplet state ${ }^{32}$ is less than $\left.1 \%\right)$. Nevertheless, 146 such low illumination conditions are quite close to the mean 147 natural solar radiation hitting the Earth surface during a sunny 148 day. In addition, by using TIR microscopy, we excite only a 149 

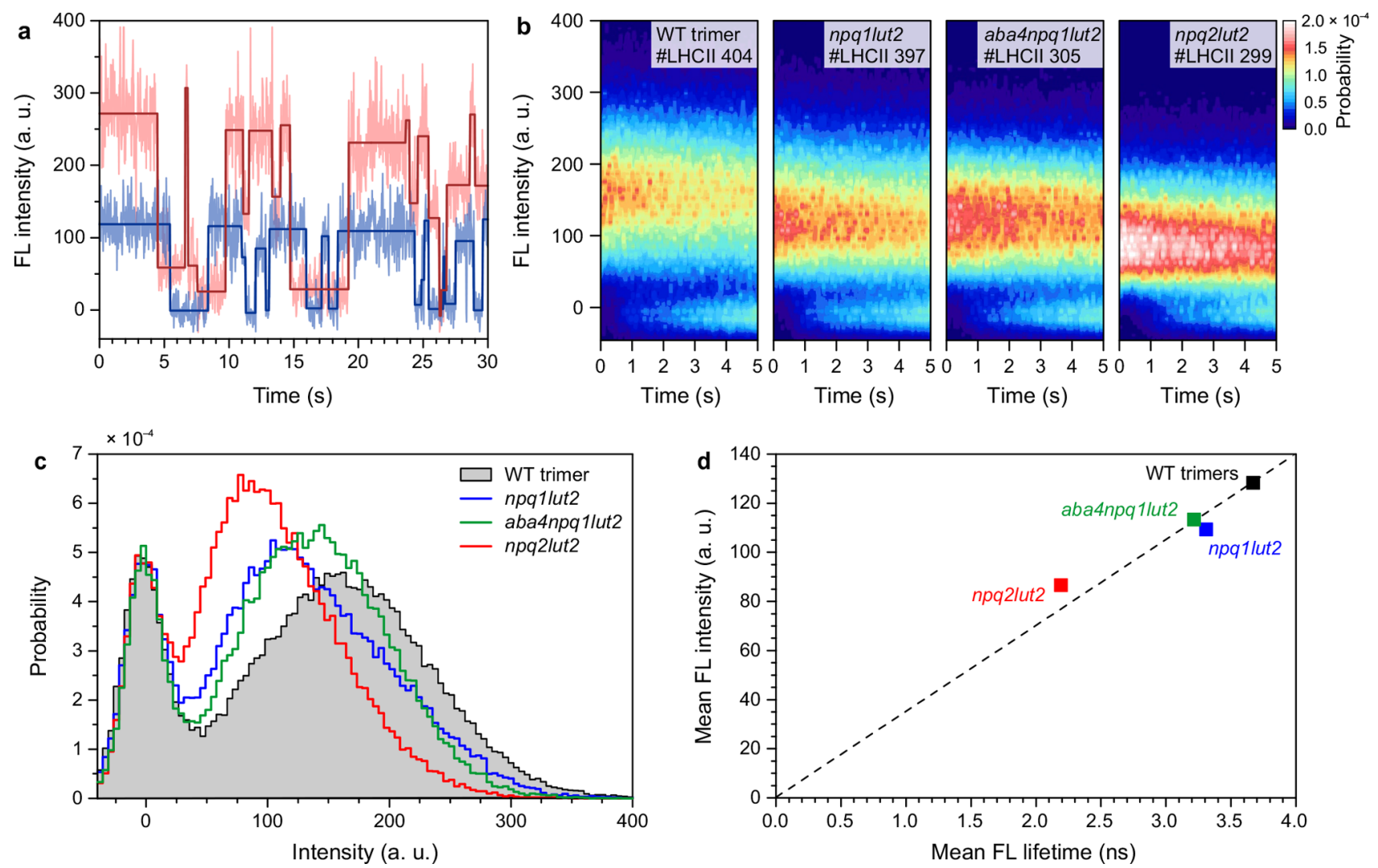

Figure 2. Fluorescence measurements in single LHCII complexes. (a) Two representative time traces of the fluorescence intensity fluctuations, measured for two different wild-type LHCII trimers (0 FL intensity level is set as the mean intensity value of the dark state). Dark step lines represent resolved intensity levels. (b) Color-coded distribution of the fluorescence intensities at different time delays, measured in WT LHCII trimers and LHCII monomers from npq1lut2, aba4npq1lut2, and npq2lut2 mutants. These distributions were acquired by collecting fluorescence time traces similar to those shown in panel (a), obtained from 300-400 (the exact number is indicated in each case) single LHCII complexes. (c) Total distribution of the resolved fluorescence intensity levels in four different LHCII samples shown in panel b. These distributions are normalized to unit area. (d) Correlation between the mean fluorescence intensities in the SM spectroscopy measurements, obtained from the FL intensity distributions shown in panel $c$, and the mean fluorescence lifetimes in the mentioned samples, obtained from the FL decay kinetics in the solubilized LHCIIs shown in Figure $1 \mathrm{~b}$.

150 small distance $(\sim 15 \mathrm{~nm})$ perpendicular to the glass coverslip, 151 which gives negligible background signal and enables much 152 better signal-to-noise ratio than confocal microscopy. Since the 153 signal from all the LHCII complexes is monitored mostly 154 simultaneously, the whole measurement takes less time and 155 guarantees that all of the measured complexes were exposed to 156 very similar conditions. And finally, TIR setup is equipped with 157 an EM-CCD camera, which has very high detection sensitivity 158 (quantum efficiency about $80 \%$ in the region of LHCII 159 emission), low noise, and excellent detection stability.

160 The acquired fluorescence signal coming from the single 161 LHCII complexes exhibited a well-known blinking behav162 ior, $^{25-30}$ when FL intensity switches quickly and reversibly 163 between several stable emission levels as a result of the 164 conformational variations of the protein scaffold ${ }^{33}$ (see Figure $1652 \mathrm{a}$ for the FL intensity fluctuations in two distinct wild-type 166 LHCII trimers). Such time traces of the fluorescence intensity 167 fluctuations were collected for 404 distinct WT LHCII trimers, 168 and the time-dependent distribution of all the obtained 169 emission levels is shown in the left panel of Figure $2 b$ as a 170 color-coded two-dimensional fluorescence map. This map 171 reveals that the intensities of the highly emitting states are 172 broadly distributed around the intensity level of $\sim 160$ a. u., 173 whereas the second much narrower peak at $\sim 0$ a. u. represents 174 nonfluorescing, or quenched, LHCII complexes (if the switching to this state was reversible) or, alternatively, the 175 complexes being completely bleached (when the switching to 176 the dark state was irreversible). Since only those single LHCII 177 complexes initially being in their light-emitting conformational 178 state were detected, there was a relatively small number of dark 179 states detected during the first second. Later on, more 180 complexes reversibly switch to the dark state, thus the zero- 181 intensity level becomes populated more often. After $\sim 25 \mathrm{~s}$ of 182 continuous illumination, the majority of the detected complexes 183 were bleached (cf. Figure S1). Only the part of the FL time 184 trace prior to photobleaching of each LHCII complex was 185 considered in any further analysis.

186

In order to compare the statistical properties of the 187 fluorescence blinking in different LHCII samples, analogous 188 measurements were also performed for LHCII monomers from 189 three lutein-deficient mutants mentioned above. The obtained 190 results are summarized in Figure $2 \mathrm{~b}$ and qualitatively resemble 191 those collected from the WT LHCII trimers. The most 192 apparent difference is the decrease of the mean FL intensity of 193 the fluorescing state in all the mutants compared to that in the 194 WT samples. This difference becomes even clearer in Figure 2c, 195 where the overall distribution of the resolved emission levels is 196 demonstrated. Interestingly, FL intensity in the LHCII 197 monomers from the mutants does not exhibit a 3-fold drop 198 compared to the wild-type LHCII trimers: while in the wild- 199 

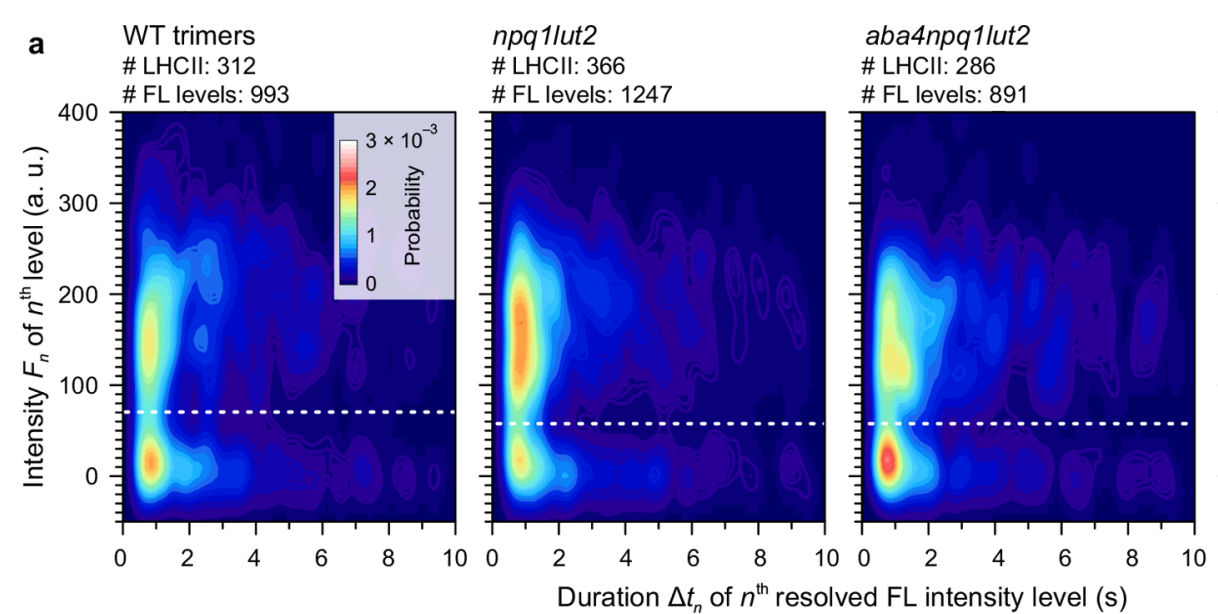

npq2lut2

\# LHCII: 289

\# FL levels: 1014

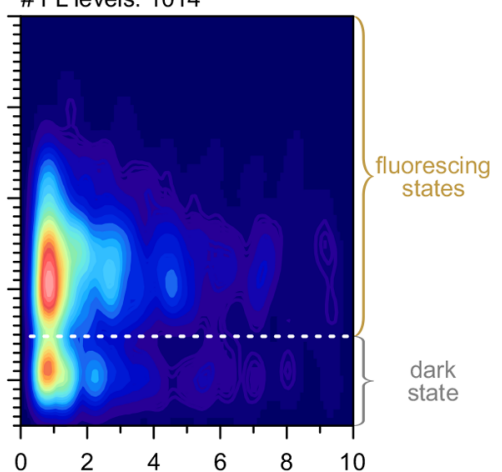

Duration $\Delta t_{n}$ of $n^{\text {th }}$ resolved FL intensity level (s)

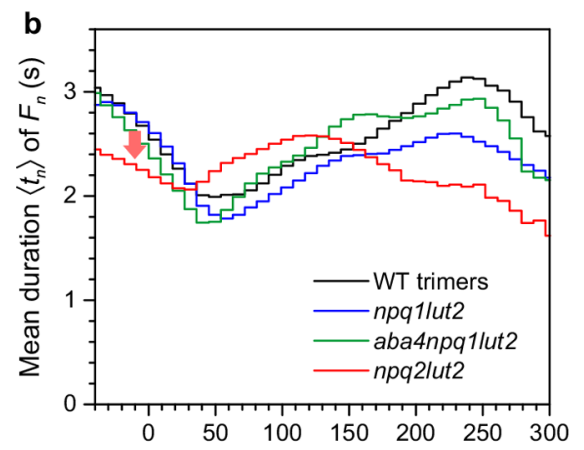

FL intensity $F_{n}$ (a. u.)
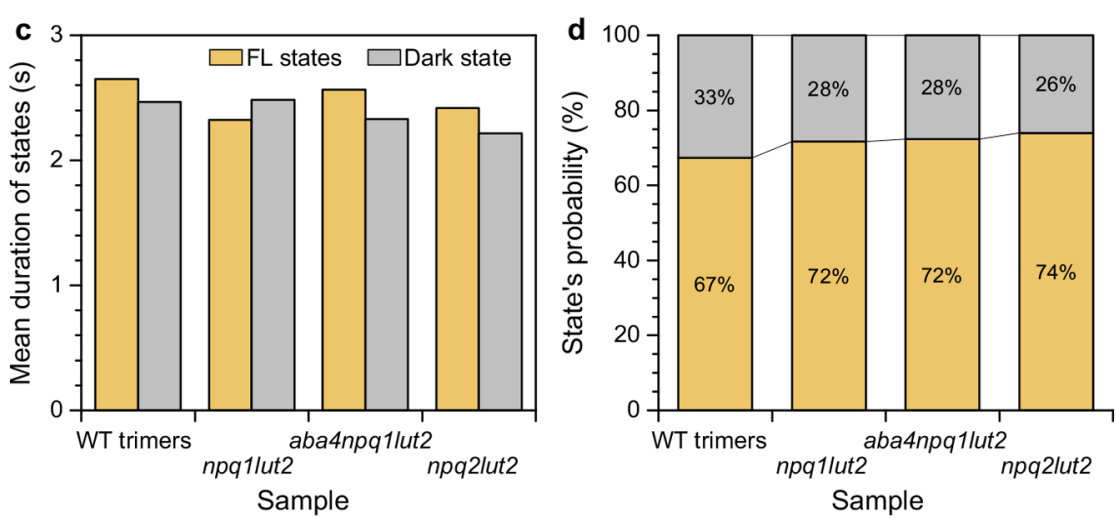

Figure 3. Correlation between the fluorescence intensity level (vertical axis) and the duration of detected states (horizontal axis). (a) Color-coded correlation maps, obtained for wild-type LHCII trimers as well as monomeric LHCIIs from the npq1lut2, aba4npq1lut2, and npq2lut2 mutants. The number of LHCII complexes analyzed and the number of the resolved FL intensity levels is indicated separately in each case. The assignment of the distinct FL intensity levels to the dark and fluorescing states is indicated with horizontal white dashed lines. (b) Mean durations of various FL intensity levels in the single LHCII complexes from different samples, obtained from the correlation maps in panel a by averaging them over horizontal axis. Red arrow indicates a notable drop in the mean duration of the quenched state in the npq2lut2 mutant. (c) Overall mean duration of the fluorescing and dark states of single LHCII complexes from various samples. (d) The relative probabilities the dark and fluorescing conformational states of various LHCII samples, obtained by integrating correlation maps in panel a over both vertical and horizontal axes within the domains separated by the white dashed lines.

200 type LHCII complexes the emission intensities of the highly 201 fluorescing states are distributed around 160 a.u., the 202 corresponding peak intensity dropped by $\sim$ one-quarter in 203 the npq1lut2 and aba4npq1lut2 mutants (115 and 130 a.u., 204 respectively). This indicates that, under our illumination 205 conditions, the probability to absorb three photons per trimer 206 while all its monomeric subunits are in the high-emitting state, 207 might be rather low (see also Methods), although the 208 possibility for trimeric and monomeric complexes to experience 209 slightly different environments (e.g., they can be differently 210 orientated during their immobilization on the PLL glass 211 coverslip) also cannot be disregarded.

212 Distributions of FL intensities in both these mutants are very 213 similar, again demonstrating that the replacement of the Neo 214 with Vio in the latter one does not significantly change the 215 overall excitation dynamics in LHCII. Meanwhile, in the LHCII 216 monomers from npq2lut2 mutant, the drop in observed FL 217 intensities was even more pronounced and exceeded $45 \%$ in 218 these complexes, and the dominating fluorescence intensity 219 decreased down to 85 a.u. In all the mutants, not only the 220 maximum position of the band of the FL intensities of the 221 strongly fluorescing states shifted toward lower intensities 222 (comparing to the WT samples), but also the amplitude of this 223 band (i.e., the number of occurrences of the corresponding FL levels) increased, while the width of this band decreased 224 accordingly. Interestingly, neither the amplitude nor the width 225 of the band corresponding to the nonfluorescing state exhibited 226 any differences between different LHCII samples, suggesting 227 the same physical origin of the dark state in all the cases. 228 Indeed, distribution of intensities around this dark state was 229 fitted with a Gaussian function, and its width within the fitting 230 error was the same as for the bleached spot intensity 231 distributions shown in Figure S1b $(20.4 \pm 1.9$ and $20.6 \pm 232$ 0.48 a.u., respectively). By taking an average of the whole 233 distribution shown in Figure 2c, including the quenched and 234 fluorescing states, the mean fluorescence intensities in different 235 samples were calculated. These mean intensities correlate with 236 the mean FL lifetimes observed during the bulk measurements, 237 as shown in Figure 2d. Thus, we can conclude that the FL 238 decay kinetics, reflecting excitation dynamics that occurs within 239 the complex on a nanosecond time scale, is strongly related to 240 the conformational dynamics of the complex as a single unit, 241 taking place on a time scale from milliseconds to several 242 seconds.

243

All four LHCII samples studied in this work exhibited a very 244 broad distribution of the intensity levels of the fluorescing 245 states. Thus, several distinct conformational states of the light- 246 harvesting complexes, each corresponding to some specific 247 

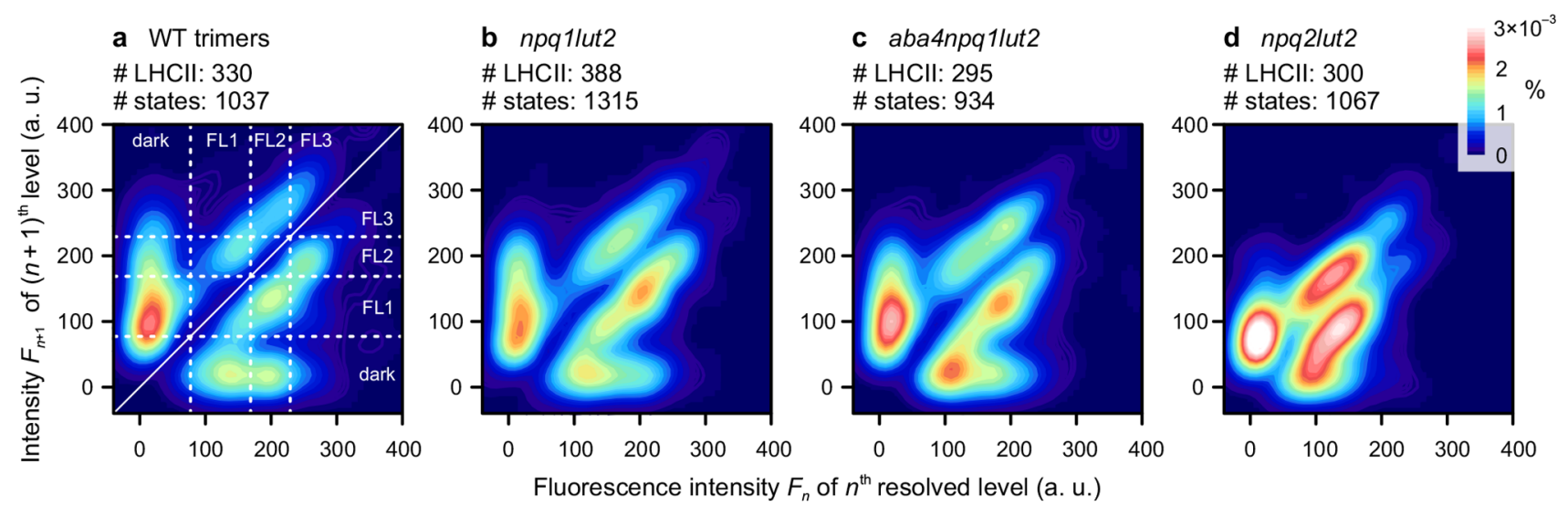

Figure 4. Transitions between different conformational states. (a) Two-dimensional transition density (TD) histogram plot for WT LHCII trimers, showing correlation between the mean intensity $F_{n}$ of $n$th state (horizontal axis) and the mean intensity $F_{n+1}$ of the following $(n+1)$ th state (vertical axis). The number of single LHCII trimers and the numbers of the resolved FL intensity levels are indicated at the top. The TD map is normalized to 1 , and the color scale on the right indicates the probability of the corresponding transition $F_{n} \rightarrow F_{n+1}$. The observed cross-peaks correspond to the conformational switching between different states of LHCII, denoted as "dark", "FL1", "FL2" and "FL3" and separated with white dashed lines. LHCII monomers from the carotenoid mutants exhibit qualitatively similar correlation maps $(b-d)$.

248 dominating fluorescence intensity, might coexist, as suggested 249 previously. $^{25,29}$ To address this issue and to characterize 250 possible conformational states, we have first evaluated the 251 correlation between each resolved FL intensity level $\left(F_{n}\right)$ and 252 the time duration $\Delta t_{n}$ that each LHCII complex was fluorescing 253 with that particular intensity before a transition to another 254 intensity level $\left(F_{n+1}\right)$ occurred. The resulting two-dimensional 255 histogram plots are shown in Figure $3 \mathrm{a}$ and demonstrate that 256 the wide distribution of the intensities of the active (highly 257 fluorescing) LHCII state in Figure $2 \mathrm{c}$ indeed has some intrinsic 258 structure. For example, in the case of WT LHCII species, we 259 can identify at least two distinct fluorescing states: one with the 260 dominating fluorescence intensity around 150 a.u. and another 261 around 230 a.u. The former state is more probable, but its 262 duration on average is shorter. Qualitatively similar correlation 263 pattern is obtained in LHCII mutants, although the dominating 264 fluorescence intensities of the bright states are reduced.

265 Most of the detected FL intensity levels had a duration from 266 several hundreds of milliseconds to $\sim 3 \mathrm{~s}$, but longer-living 267 states were also observed, in accordance with the well-known 268 power-law distribution. ${ }^{25,26}$ By averaging the correlation maps 269 in Figure 3a over the horizontal axis, we obtain the mean 270 durations $\left\langle\Delta t_{n}\left(F_{n}\right)\right\rangle$ of each resolved FL intensity level $F_{n}$, as 271 shown in Figure $3 \mathrm{~b}$. While the mean duration of the FL 272 intensity levels corresponding to the dark states (those around $273 F_{n}=0$ ) in both npq1lut2 and aba4npq1lut2 mutants remain the 274 same as in WT LHCII trimers, those in the npq2lut2 exhibit a 275 notable drop, as indicated by the red arrow in Figure $3 \mathrm{~b}$. On 276 average, the dark conformation survives for $2.5 \mathrm{~s}$ in both WT 277 trimers and npq1lut2 mutants, its mean duration then decreased 278 to $2.3 \mathrm{~s}$ in aba4npq1lut2 and further dropped down to $2.2 \mathrm{~s}$ in 279 the npq2lut 2 mutant (see Figure 3c). The description of the 280 fluorescing states is complicated by the coexistence of multiple 281 distinct conformations, thus only the mean duration of all these 282 fluorescing conformational states can be reliably evaluated: 283 while being $2.7 \mathrm{~s}$ in WT complexes, it decreases by $0.4,0.1$, and $2840.3 \mathrm{~s}$ in $n p q 1$ lut2, aba4npq1lut2, and npq2lut2, respectively. This 285 results in slightly faster conformational dynamics in all the 286 mutants compared to the wild-type LHCII trimers.

287 Finally, by integrating correlation maps shown in Figure 3a 288 over both vertical and horizontal axes within the domains corresponding to the emitting and quenched states and 289 separated by the white dashed lines, we can evaluate the 290 overall probabilities of fluorescing and dark states, as 291 demonstrated in Figure 3d. Counterintuitively, we see the 292 stabilization of the fluorescing states (manifesting itself via 57\% 293 increase in probability) in all the mutants compared to the WT 294 samples, despite the fact that the drop in the overall mean FL 295 intensity and mean excitation lifetime, observed in the mutants 296 and discussed above, would suggest an opposite effect. This 297 could indicate that replacement of luteins by either Vio or Zea 298 pigments results in more pronounced switching between 299 different fluorescing conformational states and, accordingly, 300 less frequent "visiting" of the quenched conformation. 301

To check this suggestion and to highlight the conformational 302 dynamics between different conformational states of the LHCII 303 complex, we have also calculated the correlation pattern of the 304 intensities of two detected subsequent fluorescence levels $\left(F_{n} 305\right.$ and $\left.F_{n+1}\right)$. The resulting two-dimensional transition density 306 (TD) histogram, describing probabilities of different transitions 307 between the detected states $\left(F_{n}\right)$ in the single-molecule 308 fluorescence intensity traces from WT trimeric LHCIIs, is 309 presented in Figure 4a. This TD map shows a complex $310 \mathrm{f} 4$ landscape and further reveals the hidden structure of the main 311 emission intensity band. Indeed, we can clearly distinguish 312 several cross-peaks corresponding to various transitions 313 between different conformational states: one such state is 314 readily attributed to the aforementioned dark state (with FL 315 intensity around 0 a.u.). However, we can also resolve at least 316 three distinct fluorescing states corresponding to the 317 fluorescence intensities of $\sim 100130$ a.u., $\sim 200$ a.u., and $\sim 250318$ a.u. (denoted in Figure 4a as FL1, FL2, and FL3, respectively). 319 We can also note the asymmetry between different transition 320 pathways: the most dominating transition corresponds to the 321 conformational switch of the LHCII complex from the dark 322 state into FL1 state, whereas the repopulation of the dark state 323 occurs from both FL1 and FL2 states. Somewhat less probable 324 transitions occur between FL2 and FL1 states as well as FL3 325 and FL2 states. Moreover, the elongation of the cross-peaks 326 along the main diagonal rather reveals a high level of 327 inhomogeneity of FL emission in different single LHCII 328 complexes (see also FL time traces in Figure 2a, exhibiting 329 
330 various FL intensities of the fluorescing states for distinct 331 LHCIIs).

332 The corresponding TD maps, obtained for the lutein333 deficient LHCII complexes, are shown in Figure $4 b-d$. Both 334 npq1lut2 and aba4npq1lut2 mutants exhibit very similar 335 transition pattern as the wild-type samples, except for the 336 general drop in FL intensity. However, one can also note more 337 frequent switching between distinct fluorescent states (repre338 sented by the increased amplitude of the cross-peaks at $F_{n} \approx$ 339200 and $F_{n+1} \approx 150$ a.u. and vice versa), thus supporting the 340 suggestion made above. This effect is even more pronounced in 341 the case of npq2lut2, shown in Figure 4d. In this mutant, only 342 the emitting state corresponding to the lowest FL intensity is 343 involved in the reversible transitions to/from the quenched 344 state, whereas the conformational states attributed to higher FL 345 intensity participate only in the switching to/from other 346 emitting states.

347 Based on our measurements, several conclusions regarding 348 conformational dynamics of LHCII complexes can be drawn. 349 First of all, the mean fluorescence intensity, observed during the $350 \mathrm{SM}$ measurements and related to protein conformation 351 dynamics, correlates with the time scale of fluorescence decay 352 kinetics, representing excitation energy transfer and relaxation 353 within the complex. Next, SM microscopy revealed a rather 354 high level of heterogeneity of single LHCII complexes and 355 quite complex conformational energy landscape. In particular, 356 besides the quenched state, the coexistence of at least three 357 distinct fluorescing conformational states, attributed to different $358 \mathrm{FL}$ intensity levels and experiencing strongly asymmetric 359 switching between themselves, was revealed. Each such state 360 might correspond to slightly different mutual arrangements of 361 the pigment molecules within the LHCII complex and, as 362 suggested earlier, ${ }^{29}$ result in different time scales of excitation 363 energy dynamics between the pigment molecules. Therefore, 364 partially quenched, but still fluorescing state(s) might explain 365 any nonexponentiality observed during the time-resolved 366 fluorescence measurements.

367 We have also demonstrated that the replacement of the 368 lutein pigments with either Vio or Zea, preventing the 369 formation of tightly bound trimeric complexes, results in faster 370 conformational dynamics compared to that observed in WT 371 LHCII trimers. This explains why such monomeric pigment372 protein complexes are more sensitive to a varying environment 373 such as lumen protons, as was previously observed during the in 374 vitro experiments. ${ }^{34}$ Meanwhile, the fact that all the samples 375 exhibited fluorescence blinking behavior and the observed 376 properties of the dark conformational state were rather similar 377 regardless of the carotenoid compositions reveals the identical 378 nature of the quenching mechanism in all these LHCII 379 complexes. Our observations suggest that not a particular 380 carotenoid but rather a carotenoid that is bound to a specific 381 binding locus acts as quenchers. Hence, the variability of LHCII 382 carotenoid types is less critical than their environment. This 383 result indirectly supports the model of incoherent excitation 384 energy transfer from $\mathrm{Chl}$ molecule to the optically dark short385 lived $S_{1}$ state of the available carotenoid. ${ }^{8}$ Indeed, the coherent 386 mixing of the Car and Chl excitonic states or, alternatively, the 387 formation of the $\mathrm{Car}-\mathrm{Chl}$ charge-transfer state would be very 388 sensitive to the exact position of the Car $S_{1}$ energy level (with 389 respect to the site energies of the nearby chlorophylls) and 390 therefore would lead to different results in different samples. 391 On the other hand, very broad density-of-states distribution of 392 the Car $S_{1}$ transition results in very weak effect of both the Car
$S_{1}$ site energy and small variations in the ChlCar couplings 393 (expected to take place in our different samples with various 394 carotenoid composition) on the rate of the incoherent $\mathrm{Chl} \rightarrow 395$ Car excitation transfer. ${ }^{35,36}$

396

Nevertheless, the carotenoid composition does have some 397 effect on the conformational switching of the LHCII complex 398 between different emitting states. That is not surprising since 399 carotenoids are essential structural elements of the light- 400 harvesting complexes and, as such, they can affect the overall 401 structure that, in its turn, influences the blinking behavior. 402 However, transition density patterns, shown in Figure 4, reveal 403 that the replacement of the luteins with Vio in both npq1lut2 404 and aba4npq1lut2 mutants results in very subtle structural 405 variations that do not change the overall conformational 406 dynamics of the LHCII monomer, but makes switching 407 between the strongly and moderately fluorescing states more 408 probable than in the WT samples. As a result, we observe 409 somewhat smaller mean fluorescence intensity (Figure 2c) and 410 slightly faster excitation decay kinetics (Figure $1 \mathrm{~b}$ ). Very similar 411 results, obtained for both these mutants, also confirm that the 412 Neo binding site in the $\mathrm{Chl} b$-rich peripheral side participates 413 neither in fluorescence quenching nor in the conformational 414 dynamics of the pigment protein complex as a whole. On the 415 other hand, the npq2lut2 LHCII monomers, containing Zea as 416 the only carotenoid, demonstrated even more pronounced 417 switching between fluorescing and partially quenched states, 418 which is in line with the observed nonexponentiality in 419 fluorescence decay kinetics. The qualitative differences, 420 observed for this mutant both in the bulk measurements (see 421 Figure 1b) and by means of single-molecule microscopy (see 422 Figures $3 \mathrm{~b}$ and $4 \mathrm{~d}$ ), suggest that (at lest in this mutant) the 423 mentioned partially quenching state cannot be simply the result 424 of very fast switching between the fully quenched and 425 fluorescing conformations, occurring within the binning time. 426 Rather, some additional factors should be involved. The 427 physical origin of this partially quenched conformational state 428 could be related to the formation of Chl-Zea charge transfer 429 state; the signatures of the presence of a $\mathrm{Zea}^{+}$radical cation 430 were indeed observed earlier in the Zea-enriched photo- 431 synthetic antenna. ${ }^{6,21}$ All these properties again demonstrate 432 very flexible and highly adaptable self-regulation of plants, in 433 general capable to dissipate the excess excitation energy using 434 any carotenoids available and, if needed, also being prone to 435 further fine-tuning by utilizing xanthophyll cycle to produce 436 Zea and the corresponding partially quenched conformational 437 state of the protein scaffold.

\section{METHODS}

Sample Preparation. Unstacked thylakoids were prepared from 440 $100 \mathrm{~g}$ of dark-adapted Arabidopsis thaliana leaves with the 441 midrib removed. Leaves were homogenized in $300 \mathrm{~mL}$ of icy 442 grinding medium ( 0.33 Msorbitol, $10 \mathrm{mM} \mathrm{Na} 4 \mathrm{P} 2 \mathrm{O} 7 \cdot \mathrm{H} 2 \mathrm{O}$ and 443 $130 \mathrm{mg}$ D-iso-ascorbate; $\mathrm{pH}$ 6.5) and the homogenate filtered 444 through a bilayer of muslin cloth, followed by a secondary 445 filtration through four layers of muslin interlaid with cotton 446 wool. Thylakoids were then centrifuged $(4000 \times g)$ for $10 \mathrm{~min} 447$ and the pellet gently resuspended in washing medium $(0.33 \mathrm{M} 448$ sorbitol and $10 \mathrm{mM}$ MED) before additional centrifugation. 449 The pellet was then resuspended in resuspension medium (0.33 450 $\mathrm{M}$ sorbitol, $1 \mathrm{mM}$ EDTA, $50 \mathrm{mM}$ HEPES; $\mathrm{pH}$ 7.6) and 451 osmotically shocked by mixing in $50 \mathrm{~mL}$ of break medium (10 452 $\mathrm{mM}$ HEPES; pH 7.6). After $30 \mathrm{~s}$, osmotic potential was 453 returned to normal with the addition of $50 \mathrm{~mL}$ of osmoticum 454 
455 medium (0.66 M sorbitol, $40 \mathrm{mM}$ MES; $\mathrm{pH}$ 6.5) and 456 thylakoids centrifuged $(4000 \times g)$ for $10 \mathrm{~min}$. The final pellet 457 was resuspended in resuspension medium, and aliquots were 458 frozen immediately in liquid nitrogen. The major LHCII 459 complex was isolated from unstacked Arabidopsis thaliana 460 thylakoids using isoelectric focusing. ${ }^{37}$ The protein band 461 corresponding to LHCII was collected and eluted in elution 462 buffer containing $0.01 \% \mathrm{n}$-dodecyl $\beta$-D-maltoside $(\sim 200 \mu \mathrm{M})$, 463 then followed by size exclusion purification to remove 464 ampholites (PD-10 columns, GE Healthcare). Aliquots of 465 LHCII prepared at the same concentration $(\mathrm{OD}=6)$ were 466 immediately frozen in liquid nitrogen for later use in 467 fluorescence analyses. While studying WT samples, we 468 preferred not to use any additional artificial treatments of the 469 isolated trimeric LHCII complexes, such as phospholipase, 470 which is crucial for the monomerization of the trimers. Hence, 471 we can compare the conformational dynamics in the naturally 472 occurring WT LHCII trimers with that in the naturally 473 occurring monomers of the mutant plants.

474 Streak Camera Measurements. Time-resolved fluorescence 475 dynamics of the samples were measured by means of 476 Hamamatsu C5680 streak camera with M5677 single-sweep 477 module coupled to a spectrometer. Femtosecond Yb:KGW 478 oscillator (Pharos, Light Conversion Ltd.) with a frequency 479 doubler (HIRO, Light Conversion Ltd.) producing $515 \mathrm{~nm}$ 480 sub-100 fs pulses at a $76 \mathrm{MHz}$ repetition rate was employed, 481 and a pulse picker was used to reduce the repetition rate to 20 $482 \mathrm{kHz}$ for nanosecond time scales. The beam was attenuated 483 down to about $100 \mathrm{pJ}$ per pulse and focused into about $100 \mu \mathrm{m}$ 484 spot on the sample. No intensity dependence was observed 485 when increasing the pulse energies by an order of magnitude or 486 during the measurement process. The temporal resolution of 487 the whole system was $\sim 100$ ps. All the measurements were 488 performed at $273 \mathrm{~K}$ in a fused silica cell of $0.1 \mathrm{~mm}$ optical path. 489 The obtained fluorescence decay kinetics, shown in Figure 1b, 490 were independent of the detection wavelength (see Figure S2 491 for details).

492 Single-Molecule Microscopy Measurements. Buffer Solution: 493 Buffer 1:10 mM Hepes (>99.5 Buffer grade, Carl-Roth Art.$494 \mathrm{Nr}$ : HN07.1), 1 mM MgCl2 (>99 Cell pure, Carl-Roth Art.-Nr: 495 HN78.2), $0.03 \% \mathrm{w} / \mathrm{v}$ Ÿ-DM (Lauryl- $\beta$-D-maltoside, > 99\% for 496 biochemistry, Art. CN26.2), $\mathrm{pH}=7.8$

497 Cleaning of Coverslips: Glass coverslips (Menzel-glaser \#1.5) 498 were placed in a staining jar and rinsed 3 times with ultrapure 499 water (LaboStar, Siemens). Water was exchanged with $1 \%$ 500 Alconox detergent solution (Alconox powdered precision 501 cleaner) and the jar sonicated for $10 \mathrm{~min}$ (Ultrasonic Cleaning 502 Unit RK 102H, Bandelin). Detergent solution was discarded 503 from the jar and rinsed four times with ultrapure water. Water 504 was exchanged with isopropanol (2-propanol $\geq 99.5 \%$, 505 art.no.:9866.6 Carl-Roth). Isopropanol was discarded, and the 506 jar with coverslips was vacuumed in the plasma machine (PDC507 002, Harric plasma) for $20 \mathrm{~min}$, then plasma etched at $\sim 400$ $508 \mathrm{~m}$ Torr pressure using maximal power for $5 \mathrm{~min}$.

509 Surface Modification of Coverslips and Flow Cell Assembling: 510 Clean glass coverslips were incubated with $0.01 \%$ of PLL 511 (P4707 Sigma) for $10 \mathrm{~min}$, then rinsed with Milli-Q, dried and 512 assembled into the flow cell (sticky-slide VI 0.4, 80608, IBIDI) 513 with the PLL modified side facing the sticky slide. Tubings were 514 inserted into the inlet and outlet port of the cell and channel 515 was filled with buffer1.

516 LHCII Immobilization and Imaging: $1.5 \mathrm{e}+05$ diluted LHCII $517(2 \mu \mathrm{g} / \mathrm{mL})$ dissolved in bufferl were injected into the flow cell channel and incubated for $3 \mathrm{~min} .300 \mu \mathrm{L}$ of the buffer 1 was 518 injected to wash out the excess of unbound LHCII. For 519 microscopy $100 \mu \mathrm{L}$ of buffer 1 containing 15 units $/ \mathrm{ml}$ glucose 520 oxidase (from Aspergillus niger, G6125, Sigma-aldrich), 1\% 521 glucose ( $\ddot{Y}-D-G l u c o s e, ~ G 0047, T C I$ AMERICA). Measurements 522 were performed at $23{ }^{\circ} \mathrm{C}$.

SM TIRF Microscopy: The SM fluorescence microscopy setup 524 used in this study was essentially the same as described 525 previously except for a few important improvements. ${ }^{38}$ All the 526 dichroic mirrors in the setup were replaced with $2 \mathrm{~mm}$ thick 527 TIRF flat parts glued in metal filter cubes (91032, Chroma). 528 The $635 \mathrm{~nm}$ continuous-wave laser beam is expanded 66x. The 529 laser beam was reflected off and the resulting fluorescence was 530 transmitted through a zt532/635rps-XT (Chroma) dual-band 531 dichroic mirror. The excitation light was filtered off by a 532 quadruple-band interference filter FF01-446/510/581/703 533 (Semrock). The fluorescence image was split into two spectral 534 components by T640lpxr-UF2 (Chroma) dichroic mirror. The 535 $635 \mathrm{~nm}$ excitation intensity behind the objective was $0.6 \mathrm{~mW} 536$ and exposure time was $30 \mathrm{~ms}$. In TIRF microscopy, light beam 537 after passing the objective enters the sample with the high angle 538 of incidence, so that at the interface of the regions with two 539 different refractive indexes the evanescence field is generating, 540 and technically it is difficult to measure its exact intensity. ${ }^{39} 541$ However, according to our estimations, it should be far less 542 than $1 \mathrm{~W} / \mathrm{cm}^{2}$, which corresponds to less than $\sim 3 \times 10^{18} 543$ photons per second per $\mathrm{cm}^{2}$. Hence taking into account the 544 mean absorption cross-section of the LHCII trimer, $\sigma=1.4 \times 545$ $10^{-15} \mathrm{~cm}^{2}$, 31 we conclude that the absorption rate should not 546 exceed $\sim 4500$ photons per second per LHCII trimer. $\quad 547$

In the EM-CCD camera used in this work, one detected 548 photon corresponds to $\sim 34$ counts. The signal intensity 549 (denoted in this work with a.u.) represents the amplitude of the 550 2D Gaussian fit, whose width was held constant and on average 551 was equal to $s=1.33$ pixels for all the detected spots. Therefore, 552 one detected photon corresponds to the $34 /\left(2 \pi s^{2}\right)$ a.u. $=3$ a.u. 553

Data Analysis: All data analysis procedures were performed 554 and graphs prepared in Igor Pro (Wavemetrics) program using 555 a custom written analysis package (available upon direct request 556 to the author or under the link: http://www.igorexchange.com/ 557 project/TEA_MT). To detect immobilized LHCII, fluorescent 558 spots were detected in images acquired using $635 \mathrm{~nm}$ laser. To 559 make this detection more reliable, several frames from the 560 beginning of the image series were averaged. From this 561 averaged image a filtered image, enhancing the fluorescent 562 spots, was generated and converted into a fluorescent spot 563 probability image. ${ }^{40}$ Spots having the probability above the 564 manually defined value were fitted to the $2 \mathrm{D}$ symmetrical 565 Gaussian to extract precise center position and width. Spots 566 having higher than manually defined fitting error and center 567 positions closer than 3 pixels were rejected. Intensity-versus- 568 time dependency was extracted for all accepted spots from the 569 series of images acquired at $635 \mathrm{~nm}$ excitation using 2D 570 symmetrical Gaussian fit (center position and width was held 571 constant). SM intensity was expressed by an amplitude of the 572 2D Gaussian fit. When LHCII switches into the dark state or 573 bleaches out, the intensity of the local background becomes 574 similar or sometimes even higher than in the center of the 575 previously fluorescing spot. Therefore, sometimes intensity that 576 we record becomes negative (i.e., the amplitude of the 2D 577 Gaussian becomes less than 0). Hence in Figures 2-4, the 0578 a.u. level can be treated as the mean intensity of the dark state. 579 Finally, manual check and selection of those signals showing 580 
581 characteristic single-molecule features (typical single-molecule 582 fluorescence intensity and a single bleaching step) was 583 performed. Only that selected data was used to make plots. 584 SM fluorescence intensity traces were idealized using a custom585 made intensity change point (ICP) detection algorithm without 586 clustering. This algorithm is similar to a previously published 587 method, $^{25}$ except that amplitude of the ICP is a constant and 588 predefined by the user. Basically, it scans a trace point-by-point 589 with an 8 point window, takes an average of the first 4 points 590 and the last 4 points within the window and calculates a step 591 amplitude (the difference between the averages) that is then 592 compared with some threshold value. The latter was found 593 empirically by testing different numerical values. Value of 40 594 a.u. for WT LHCII was high enough not to find any steps in the 595 bleached part of the signal and low enough not to miss any 596 significant steps in the active part of the signal. Next, we 597 calculated step amplitude thresholds for mutant samples by 598 multiplying the step amplitude of WT LHCII with a ratio 599 between mean fluorescence intensity of WT and each of the 600 mutant samples (Figure $2 \mathrm{~d}$ ). For example, for the npq1lut2 and 601 aba4npq1lut2 mutants, $40 /(125 / 110)=35$ a.u., and for $602 n p q 2$ lut $2,40 /(125 / 80)=25$ a.u. In the case where the step 603 amplitude is higher than this threshold value, the center 604 position of this window is recorded as a putative ICP. To be 605 accepted, an ICP has to fulfill the criteria for the durations and 606 slopes of the states it separates. Both states have to be not 607 shorter than half of the scanning window length. The sum of 608 the absolute slope values of the line fits of the states has to be 609 smaller than a set value of 10 .

\section{ASSOCIATED CONTENT}

\section{S Supporting Information}

612 The Supporting Information is available free of charge on the 613 ACS Publications website at DOI: 10.1021/acs.jpclett.7b02634.

614 Biexponential description of the fluorescence decay 615 kinetics shown in Figure $1 \mathrm{~b}$, distributions of the 616 fluorescence intensities at different time delays, demon617 strating the bleaching of the observed single LHCII 618 complexes, and fluorescence decay kinetics in various 619 samples, detected at different wavelengths during the 620 bulk streak camera measurements (PDF)

\section{AUTHOR INFORMATION}

\section{Corresponding Author}

623 *E-mail: leonas.valkunas@ff.vu.lt.

624 ORCID

625 Marijonas Tutkus: 0000-0002-5795-1347

626 Jevgenij Chmeliov: 0000-0002-7591-1373

627 Danielis Rutkauskas: 0000-0003-4705-2222

\section{Notes}

629 The authors declare no competing financial interest.

\section{ACKNOWLEDGMENTS}

631 We would like to acknowledge Dr. Petra Ungerer for providing 632 the samples, Egidijus Songaila for the streak-camera fluo633 rescence measurements, and Oskaras Venckus for help with the 634 data analysis. J.C. and L.V. were supported by the Research 635 Council of Lithuania (LMT Grant No. MIP-080/2015). A.R. 636 was supported by The Royal Society Wolfson Research Merit 637 Award and U.K. BBSRC Grant BB/L019027/1.

\section{REFERENCES}

638

(1) van Amerongen, H.; Valkunas, L.; van Grondelle, R. Photo- 639 synthetic Excitons; World Scientific: Singapore, 2000; p 590.

(2) Blankenship, R. E. Molecular Mechanisms of Photosynthesis, 2nd 641 ed.; Wiley Blackwell: Chichester, U.K., 2014; p 296 p.

(3) Fleming, G. R.; Schlau-Cohen, G. S.; Amarnath, K.; Zaks, J. 643 Design Principles of Photosynthetic Light-Harvesting. Faraday Discuss. 644 2012, 155, 27-41.

645

(4) Croce, R.; van Amerongen, H. Natural Strategies for Photo- 646 synthetic Light Harvesting. Nat. Chem. Biol. 2014, 10, 492-501. 647 (5) Ruban, A. V.; Johnson, M. P.; Duffy, C. D. P. The 648 Photoprotective Molecular Switch in the Photosystem II Antenna. 649 Biochim. Biophys. Acta, Bioenerg. 2012, 1817, 167-181.

650

(6) Holt, N. E.; Zigmantas, D.; Valkunas, L.; Li, X. P.; Niyogi, K. K.; 651 Fleming, G. R. Carotenoid Cation Formation and the Regulation of 652 Photosynthetic Light Harvesting. Science 2005, 307, 433-436. 653

(7) Pascal, A. A.; Liu, Z. F.; Broess, K.; van Oort, B.; van Amerongen, 654 H.; Wang, C.; Horton, P.; Robert, B.; Chang, W. R.; Ruban, A. 655 Molecular Basis of Photoprotection and Control of Photosynthetic 656 Light-Harvesting. Nature 2005, 436, 134-137.

(8) Ruban, A. V.; Berera, R.; Ilioaia, C.; van Stokkum, I. H. M.; 658 Kennis, J. T. M.; Pascal, A. A.; van Amerongen, H.; Robert, B.; Horton, 659 P.; van Grondelle, R. Identification of a Mechanism of Photoprotective 660 Energy Dissipation in Higher Plants. Nature 2007, 450, 575-578. 661

(9) Ahn, T. K.; Avenson, T. J.; Ballottari, M.; Cheng, Y. C.; Niyogi, K. 662 K.; Bassi, R.; Fleming, G. R. Architecture of a Charge-Transfer State 663 Regulating Light Harvesting in a Plant Antenna Protein. Science 2008, 664 320, 794-797.

(10) Staleva, H.; Komenda, J.; Shukla, M. K.; Šlouf, V.; Kaňa, R.; 666 Polívka, T.; Sobotka, R. Mechanism of Photoprotection in the 667 Cyanobacterial Ancestor of Plant Antenna Proteins. Nat. Chem. Biol. 668 2015, 11, 287-291.

669

(11) Chmeliov, J.; Gelzinis, A.; Songaila, E.; Augulis, R.; Duffy, C. D. 670 P.; Ruban, A. V.; Valkunas, L. The Nature of Self-Regulation in 671 Photosynthetic Light-Harvesting Antenna. Nat. Plants 2016, 2, 16045. 672

(12) Allen, J. F. Protein Phosphorylation in Regulation of 673 Photosynthesis. Biochim. Biophys. Acta, Bioenerg. 1992, 1098, 275-335. 674 (13) Belgio, E.; Kapitonova, E.; Chmeliov, J.; Duffy, C. D. P.; 675 Ungerer, P.; Valkunas, L.; Ruban, A. V. Economic Photoprotection in 676 Photosystem II That Retains a Complete Light-Harvesting System 677 with Slow Energy Traps. Nat. Commun. 2014, 5, 4433.

(14) Liu, Z. F.; Yan, H. C.; Wang, K. B.; Kuang, T. Y.; Zhang, J. P.; 679 Gui, L. L.; An, X. M.; Chang, W. R. Crystal Structure of Spinach Major 680 Light-Harvesting Complex at $2.72 \AA$ Resolution. Nature 2004, 428, 681 287-292.

682

(15) Bishop, N. I. The $\beta, \varepsilon$-Carotenoid, Lutein, is Specifically 683 Required for the Formation of the Oligomeric Forms of the Light 684 Harvesting Complex in the Green Alga,. J. Photochem. Photobiol., B 685 1996, 36, 279-283.

686

(16) Fuciman, M.; Enriquez, M. M.; Polívka, T.; Dall'Osto, L.; Bassi, 687 R.; Frank, H. A. Role of Xanthophylls in Light Harvesting in Green 688 Plants: A Spectroscopic Investigation of Mutant LHCII and Lhcb 689 Pigment-Protein Complexes. J. Phys. Chem. B 2012, 116, 3834-3849. 690 (17) Niyogi, K. K.; Shih, C.; Chow, W. S.; Pogson, B. J.; DellaPenna, 691 D.; Björkman, O. Photoprotection in a Zeaxanthin- and Lutein- 692 Deficient Double Mutant of Arabidopsis. Photosynth. Res. 2001, 67, 693 139-145.

694

(18) Bode, S.; Quentmeier, C. C.; Liao, P.-N.; Hafi, N.; Barros, T.; 695 Wilk, L.; Bittner, F.; Walla, P. J. On the Regulation of Photosynthesis 696 by Excitonic Interactions between Carotenoids and Chlorophylls. Proc. 697 Natl. Acad. Sci. U. S. A. 2009, 106, 12311-12316.

(19) Müller, M. G.; Lambrev, P.; Reus, M.; Wientjes, E.; Croce, R.; 699 Holzwarth, A. R. Singlet Energy Dissipation in the Photosystem II 700 Light-Harvesting Complex Does Not Involve Energy Transfer to 701 Carotenoids. ChemPhysChem 2010, 11, 1289-1296.

(20) Ware, M. A.; Dall'Osto, L.; Ruban, A. V. An In Vivo 703 Quantitative Comparison of Photoprotection in Arabidopsis Xantho- 704 phyll Mutants. Front. Plant Sci. 2016, 7, 841. 
706 (21) Dall'Osto, L.; Cazzaniga, S.; Bressan, M.; Paleček, D.; Židek, K.; 707 Niyogi, K. K.; Fleming, G. R.; Zigmantas, D.; Bassi, R. Two 708 Mechanisms for Dissipation of Excess Light in Monomeric and 709 Trimeric Light-Harvesting Complexes. Nat. Plants 2017, 3, 17033.

710 (22) Tian, L.; Xu, P.; Chukhutsina, V. U.; Holzwarth, A. R.; Croce, R.

711 Zeaxanthin-Dependent Nonphotochemical Quenching Does Not 712 Occur in Photosystem I in the Higher Plant. Proc. Natl. Acad. Sci. U. 713 S. A. 2017, 114, 4828-4832.

714 (23) Havaux, M.; Dall'Osto, L.; Cuiné, S.; Giuliano, G.; Bassi, R. The 715 Effect of Zeaxanthin as the Only Xanthophyll on the Structure and 716 Function of the Photosynthetic Apparatus in Arabidopsis thaliana. J. 717 Biol. Chem. 2004, 279, 13878-13888.

718 (24) Tutkus, M.; Marciulionis, T.; Sasnauskas, G.; Rutkauskas, D. 719 DNA-Endonuclease Complex Dynamics by Simultaneous FRET and 720 Fluorophore Intensity in Evanescent Field. Biophys. J. 2017, 112, 850721858 .

722 (25) Krüger, T. P. J.; Ilioaia, C.; van Grondelle, R. Fluorescence 723 Intermittency from the Main Plant Light-Harvesting Complex: 724 Resolving Shifts between Intensity Levels. J. Phys. Chem. B 2011, 725 115, 5071-5082.

726 (26) Krüger, T. P. J.; Ilioaia, C.; Valkunas, L.; van Grondelle, R. 727 Fluorescence Intermittency from the Main Plant Light-Harvesting 728 Complex: Sensitivity to the Local Environment. J. Phys. Chem. B 2011, $729115,5083-5095$.

730 (27) Krüger, T. P. J.; Wientjes, E.; Croce, R; van Grondelle, R. 731 Conformational Switching Explains the Intrinsic Multifunctionality of 732 Plant Light-Harvesting Complexes. Proc. Natl. Acad. Sci. U. S. A. 2011, 733 108, 13516-13521.

734 (28) Krüger, T. P. J.; Ilioaia, C.; Johnson, M. P.; Ruban, A. V.; 735 Papagiannakis, E.; Horton, P.; van Grondelle, R. Controlled Disorder 736 in Plant Light-Harvesting Complex II Explains Its Photoprotective 737 Role. Biophys. J. 2012, 102, 2669-2676.

738 (29) Schlau-Cohen, G. S.; Yang, H.-Y.; Krüger, T. P. J.; Xu, P.; 739 Gwizdala, M.; van Grondelle, R.; Croce, R.; Moerner, W. E. Single740 Molecule Identification of Quenched and Unquenched States of 741 LHCII. J. Phys. Chem. Lett. 2015, 6, 860-867.

742 (30) Kondo, T.; Pinnola, A.; Chen, W. J.; Dall'Osto, L.; Bassi, R.; 743 Schlau-Cohen, G. S. Single-Molecule Spectroscopy of LHCSR1 744 Protein Dynamics Identifies Two Distinct States Responsible for 745 Multi-Timescale Photosynthetic Photoprotection. Nat. Chem. 2017, 9, $746772-778$.

747 (31) Krüger, T. P. J.; Novoderezhkin, V. I.; Ilioaia, C.; van Grondelle, 748 R. Fluorescence Spectral Dynamics of Single LHCII Trimers. Biophys. 749 J. 2010, 98, 3093-3101.

750 (32) Gruber, J. M.; Chmeliov, J.; Krüger, T. P. J.; Valkunas, L.; van 751 Grondelle, R. Singlet-Triplet Annihilation in Single LHCII Complexes. 752 Phys. Chem. Chem. Phys. 2015, 17, 19844-19853.

753 (33) Gwizdala, M.; Berera, R.; Kirilovsky, D.; Van Grondelle, R.; 754 Krüger, T. P. Controlling Light Harvesting with Light. J. Am. Chem. 755 Soc. 2016, 138, 11616-11622.

756 (34) Ruban, A. V.; Young, A. J.; Horton, P. Dynamic Properties of 757 the Minor Chlorophyll $a / b$ Binding Proteins of Photosystem II, an in 758 vitro Model for Photoprotective Energy Dissipation in the Photo759 synthetic Membrane of Green Plants. Biochemistry 1996, 35, 674-678. 760 (35) Balevičius, V.; Gelzinis, A.; Abramavicius, D.; Mančal, T.; 761 Valkunas, L. Excitation Dynamics and Relaxation in a Molecular 762 Heterodimer. Chem. Phys. 2012, 404, 94-102.

763 (36) Fox, K. F.; Balevičius, V.; Chmeliov, J.; Valkunas, L.; Ruban, A. 764 V.; Duffy, C. D. P. The Carotenoid Pathway: What is Important for 765 Excitation Quenching in Plant Antenna Complexes? Phys. Chem. 766 Chem. Phys. 2017, 19, 22957-22968.

767 (37) Ruban, A. V.; Young, A. J.; Pascal, A. A.; Horton, P. The Effects 768 of Illumination on the Xanthophyll Composition of the Photosystem 769 II Light-Harvesting Complexes of Spinach Thylakoid Membranes. 770 Plant Physiol. 1994, 104, 227-234.

771 (38) Rutkauskas, D.; Petkelyte, M.; Naujalis, P.; Sasnauskas, G.; 772 Tamulaitis, G.; Zaremba, M.; Siksnys, V. Restriction Enzyme Ecl18kI773 Induced DNA Looping Dynamics by Single-Molecule FRET. J. Phys. 774 Chem. B 2014, 118, 8575-8582.
(39) Martin-Fernandez, M.; Tynan, C.; Webb, S. A 'Pocket Guide' 775 To Total Internal Reflection Fluorescence. J. Microsc. 2013, 252, 16- 776 22.

(40) Yang, L.; Parton, R.; Ball, G.; Qiu, Z.; Greenaway, A. H.; Davis, 778 I.; Lu, W. An Adaptive Non-Local Means Filter for Denoising Live- 779 Cell Images and Improving Particle Detection. J. Struct. Biol. 2010, 780 $172,233-243$. 